Research Article

\title{
Identification of a novel COL1A1 frameshift mutation, c.700delG, in a Chinese osteogenesis imperfecta family
}

\author{
Xiran Wang ${ }^{1,3^{*}}, \mathrm{Yu} \mathrm{Pei}^{1^{*}}$, Jingtao Dou${ }^{1}$, Juming $\mathrm{Lu}^{1}$, Jian $\mathrm{Li}^{2}$ and Zhaohui $\mathrm{Lv}^{1}$ \\ ${ }^{1}$ Department of Endocrinology, Chinese PLA General Hospital, Beijing, China. \\ ${ }^{2}$ Department of Elderly Endocrinology, Chinese PLA General Hospital, Beijing, China. \\ ${ }^{3}$ Department of Cadre's Ward, The Second Artillery General Hospital Chinese PLA, Beijing, China.
}

\begin{abstract}
Osteogenesis imperfecta $(\mathrm{OI})$ is a family of genetic disorders associated with bone loss and fragility. Mutations associated with OI have been found in genes encoding the type I collagen chains. People with OI type I often produce insufficient $\alpha 1$-chain type I collagen because of frameshift, nonsense, or splice site mutations in COL1A1 or COL1A2. This report is of a Chinese daughter and mother who had both experienced two bone fractures. Because skeletal fragility is predominantly inherited, we focused on identifying mutations in COL1A1 and COL1A2 genes. A novel mutation in COL1A1, c.700delG, was detected by genomic DNA sequencing in the mother and daughter, but not in their relatives. The identification of this mutation led to the conclusion that they were affected by mild OI type I. Open reading frame analysis indicated that this frameshift mutation would truncate $\alpha 1$-chain type I collagen at residue p263 (p.E234KfsX264), while the wild-type protein would contain 1,464 residues. The clinical data were consistent with the patients' diagnosis of mild OI type I caused by haploinsufficiency of $\alpha 1$-chain type I collagen. Combined with previous reports, identification of the novel mutation COL1A1-c.700de/G in these patients suggests that additional genetic and environmental factors may influence the severity of OI.
\end{abstract}

Keywords: Osteogenesis imperfecta, Chinese OI type 1 family, type I collagen, sequence analysis, frameshift mutation.

Received: December 1, 2013; Accepted: August 9, 2014.

\section{Introduction}

Osteogenesis imperfecta (OI), also known as brittle bone disease (Marini et al., 2007), is a group of rare heritable connective tissue disorders characterized by skeletal fractures with mild trauma, secondary deformities, and extraskeletal manifestations (Ben Amor et al., 2011; Marini et al., 2007). Such manifestations may include blue sclerae, progressive hearing loss, dentinogenesis imperfecta, and hyperlaxity of ligaments and skin (Ben Amor et al., 2011; Marini et al., 2007). The prevalence of OI ranges from 1 in 10,000 to 1 in 25,000 (Byers and Cole, 2002).

OI was originally classified into four clinical types, I-IV, based on its clinical features and radiographs (Sillence et al., 1979; Table S1). Type V has been associated with hyperplastic callus formation (Glorieux et al., 2000), type VI with a mineralization defect (Glorieux et al., 2002) and type VII with a range of severity that may be lethal (Ward et al., 2002). OI types VIII and IX are usually lethal (Barnes et al., 2012; Moul et al., 2013). OI severity ranges from intrauterine fractures and perinatal lethality to ex-

Correspondence to Jingtao Dou. Department of Endocrinology, Chinese PLA General Hospital, 28 Fuxing Road, Haidian district, Beijing 100853, China. E-mail: jingtaodou@163.com.

${ }^{*}$ These authors contributed equally to this article. tremely mild forms without fractures (Ben Amor et al., 2011). The severity of bone fragility of OI patients is highest in types VIII and IX, and declines progressively from type II to type VII. Type I exhibits the least severe fragility (Rauch and Glorieux, 2005). Most OI types (I-VI) show autosomal dominant inheritance, but two of the rarer types (VII and VIII) exhibit autosomal recessive disorders (Ward et al., 2002; Ben Amor et al., 2011; Table S1).

Type I collagen fibers contain three polypeptide chains. The vast majority of fibrils are composed of two $\alpha 1$ and one $\alpha 2$ chains, but a small number are composed of three $\alpha 1$ chains in a triple helix structure. The translated collagen $\alpha$ chains include propeptides at each end, one at the amino terminus end and one at the carboxyl terminus end. These propeptides help to connect and arrange the three chains which subsequently interwine in a zipper-like fashion into the triple helix configuration.

Most patients with types I-IV OI (90\%) have a mutation in one of the two collagen type I genes, COL1A1 or COL1A2 (Ben Amor et al., 2011; van Dijk et al., 2012; Table S1). COL1A1 extends $18 \mathrm{~kb}$ from $17 \mathrm{q} 21.33$ to $17 \mathrm{q} 22$ and has 52 exons. COL1A2 extends $37 \mathrm{~kb}$ from $7 \mathrm{q} 21.3$ to $7 \mathrm{q} 22.3$ and has 52 exons. More than 200 mutations in collagen 1 genes have been associated with OI (Ben Amor et al., 
2011). These mutations occur in most exons and introns of COL1A1 and COL1A2 (http://www.le.ac.uk/ge/collagen/; Dalgleish, 1997, 1998).

Many OI type I patients have COL1A1 and COL1A2 frameshift mutations, splice site mutations, and insertion mutations resulting in haploinsufficiency, or the expression of approximately half the amount of collagen type I mRNA and protein, leading to weaker bones (Redford-Badwal et al., 1996; Slayton et al., 2000; Peng et al., 2012). Other deletions, insertions, and point mutations in COL1A1 or COL1A2 induce conformational changes in the collagen $\alpha 1$ and $\alpha 2$ chains and lead to knot microfibril structure disorders, altered deposition of hydroxyapatite, brittleness, reduced resistance to external force, altered stability of the collagen triple helix, and fragility (Pallos et al., 2001; Witecka et al., 2008; Ben Amor et al., 2011).

Clinical diagnosis of osteogenesis imperfecta is straightforward in individuals with a positive family history and several typical OI features (van Dijk et al., 2012). However, despite the presence of several physical manifestations, some patients are difficult to diagnose in the absence of an affected family member and without apparent association between bone frailty and obvious extraskeletal abnormalities. In these cases, analyses of type I collagen genes for mutations can provide helpful information since $90 \%$ of OI-associated mutations arise in collagen I (van Dijk et al., 2012).

Here, we present the results of COL1A1 and COL1A2 sequencing analysis from DNA samples collected from two related Chinese patients with several typical features of OI. A novel mutation not previously associated with COL1A1 was detected. These results will help to provide a better diagnosis of mild OI type I when physical symptoms are mild.

\section{Materials and Methods}

\section{Patients}

Two related Han Chinese patients, mother and daughter, presented with blue sclerae, bone pain, and dentinogenesis imperfecta. Each had experienced two bone fractures. We reviewed their clinical features, medical history, physical examination, radiologic images, and biochemical parameters, and diagnosed them with type I osteogenesis imperfecta. Both affected family members, unaffected family members (maternal grandmother, father, and two sisters), and controls agreed to a genetic analysis and provided informed consent for the study approved by the Endocrinology Department of General Hospital of PLA. Their pedigree is shown in Figure 1.

\section{Genetic analysis}

Blood samples were obtained from the two patients, their family members, and 50 ethnically matched, unrelated controls. Genomic DNA was isolated from peripheral leu-

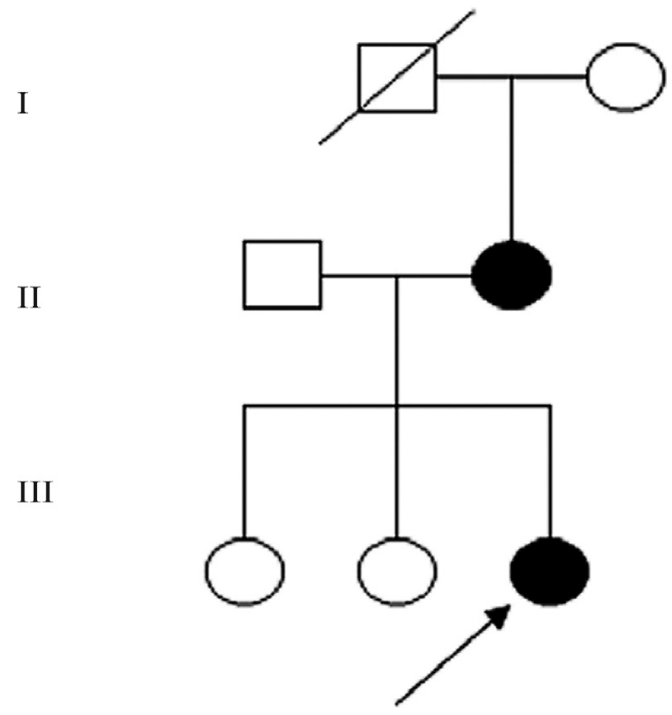

Figure 1 - The osteogenesis imperfecta pedigree of the nuclear family of the proband.

kocytes by using a whole blood DNA extraction kit (Qiagen Co., Valencia, CA) according to the manufacturer's instructions. PCR was performed to detect sequences in all exons and exon-intron boundaries of COL1A1 and COL1A2. Fifty-seven pairs of primers, 17 for COL1A1 and 40 for COL1A2 (sequences available upon request), were designed and verified with Primer 5 software (Softonic, Shanghai, China) using the genomic sequences of COL1A1 (GenBank accession NG_007400.1) and COL1A2 (Genbank accession NG_007405.1).

DNA sequence analysis was performed by automated sequencing in an ABI genetic analyzer (Model 377; ABI, USA) by the Ying Jun Gene Company (Shanghai, China). Sequencing results were compared with the GenBank database and confirmed through sequencing of clones. DNA was cloned into a PGEM-T Easy Vector following the manufacturer's instructions (Promega, Madison, WI). The osteogenesis imperfecta and Ehlers-Danlos syndrome variant databases compiled of previously reported OI mutations in COL1A1 and COL1A2, the 1000 Genomes database, and the UniProKB database were also used for comparison. PCR and sequencing of COL1A1 and COL1A2 genes in samples of other family members were performed with the same methods.

Open reading frame analysis of the mutated sequence was performed by the ORF Finder, part of the BLAST software suite. The parental protein sequence and the protein sequence corresponding to the c.700delG mutation were aligned using the align function of BLAST software (NCBI). The 1000 Genomes database was also used to search for the presence of this mutation by using the alignment function of the BLAST software and the following search sequence: 5'-TTTTCTCTCCCTCTCAGGGGA AGCTGGAAAACCTGGT CGTCCTGGTGAGCG-3'. 


\section{Results}

\section{Patient description}

The proband was a 13-year-old girl of Han descent from the Shanxi province of China. Her spontaneous fullterm delivery was uneventful. The proband began walking at 15 months. She had suffered two bone fractures in the long bone and vertebra. The first fracture occurred in the right tibia/fibula after a slight injury at 16 months old and its healing was achieved with fixation of a plate. The second fracture was caused by a slight hit to the abdomen. The proband did not grow in height during the last two years. Mental development was normal. Physical examination revealed short stature, blue sclerae, discrete signs of dentinogenesis imperfecta, pigeon breast, deformities of slight scoliokyphosis and joint hypermobility. X-rays showed thoracic-lumbar vertebra compression fracture and osteoporosis, acetabulum, and femoral head protrusion acetabulum into the pelvis.

The mother is a 43-year-old of Han Chinese descent. She had two fractures in childhood: one in the right tibia/fibula that occurred at age 5 and the other in the right radius at age 8 . She presented with hearing loss that had onset near the age of 20. Physical examination revealed blue sclerae, discrete signs of dentinogenesis imperfecta, deformity of scoliosis, and joint hypermobility. Blood samples were analyzed from the two patients and blood serum calcium, phosphorus, and parathyroid hormone levels were all within the normal range (data not shown).

\section{Mutation analysis}

Sequence analysis revealed no mutations in the COL1A2 gene from the proband or her mother. However, sequence analysis of COL1A1 identified a heterozygous frameshift mutation in exon 10 from both subjects at nucleotide 700 of the mRNA, named as c.700delG, with nucleotide 1 defined as A of the initiating ATG (Figure 2). This mutation was not identified in other family members (two sisters, father, and maternal grandmother of the proband) or ethnically matched controls. Importantly, a comparison with the compiled list of COL1A1 mutations associated with OI in the osteogenesis imperfecta and Ehlers-Danlos syndrome variant databases (Dalgleish, 1997, 1998 and http://www.uniprot.org/uniprot/P02452a) revealed that the mutation is novel and had not been previously reported.

This novel, heterozygous c.700delG frameshift mutation in COL1A1 was inherited in an autosomal dominant manner and was correlated with OI symptoms (Figure 2). An open reading frame (ORF) analysis indicated that translation of the COL1A1-c.700delG mRNA would initiate at the standard methionine and would be $100 \%$ homologous until amino acid 233. However, the mutation would produce distinct amino acids from p234 to p263 when compared to wild-type collagen $\alpha 1$ and would terminate at the

a) Wild type

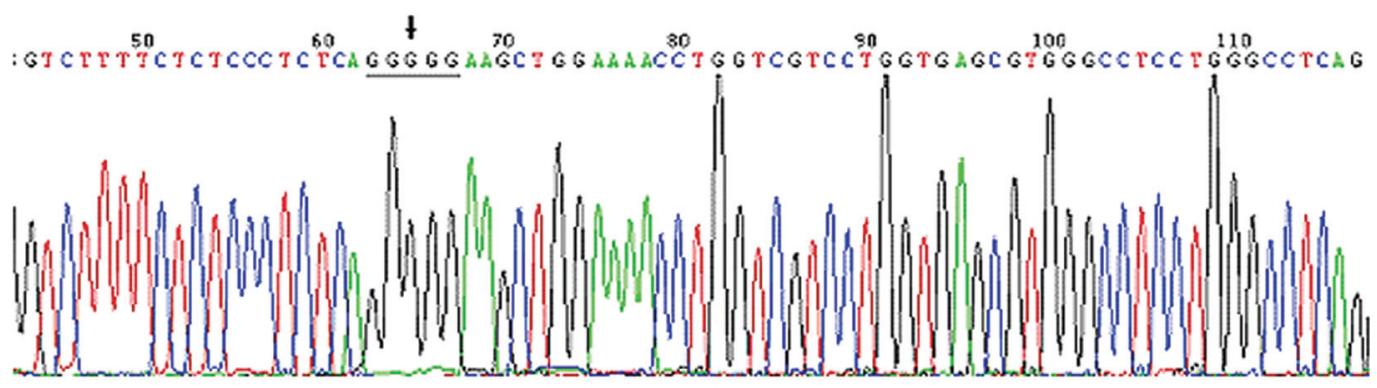

b) Proband

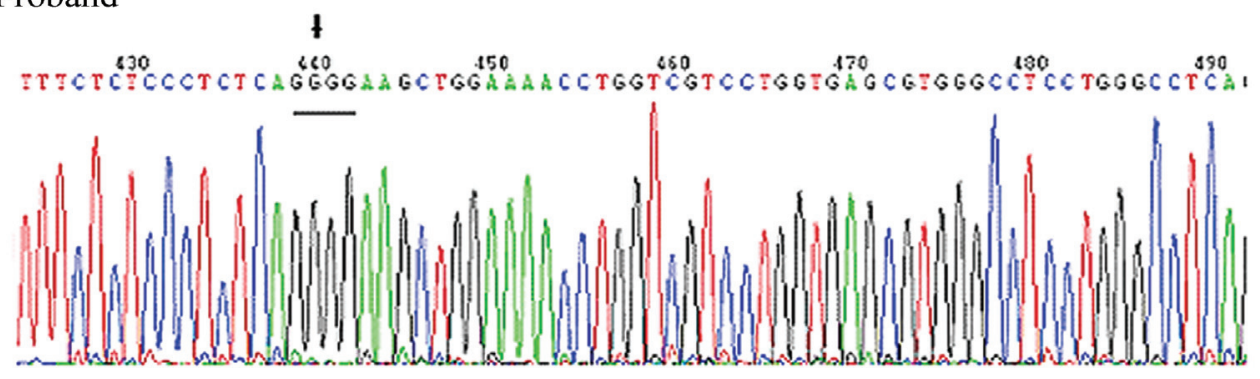

Figure 2 - Sequence analysis of COL1A1 from a wild-type control and the same DNA region from the proband containing the mutation. DNA sequencing was initiated in the ninth intron and extended into the tenth exon. (A) Genomic DNA sequence of the control wild-type allele. (B) DNA sequence of a COL1A1 clone from the proband. The DNA sequence of the same region from the mother was identical to the proband (data not shown). 
UGA stop codon at nucleotide 792 in exon 10 (Figure 3). This COL1A1 mutation would be named p.E234KfsX264. According to the ORF analysis, the putative 263 amino acid collagen $\alpha 1700 \mathrm{delG}$ mutation would contain the signal peptide (p1-22), propeptide (p23-161) and the first 102 residues of the main collagen $\alpha 1$ chain (p162-1218 and the triple helical region from p179-1192), but no C-terminal propeptide ( $\mathrm{p} 1219-1464)$. Thus, the mutation produces a truncated form of COL1A1. The second allele was normal in the proband and mother. The mutation was not described in genomes submitted to the 1000 Genomes database.

\section{Discussion}

Mutations in COL1A1 and COL1A2 account for approximately $90 \%$ of OI cases and usually occur in patients with OI types I-IV. OI symptoms can also occur in patients with mutations in genes that code proteins that interact with type I collagen, including cartilage associated protein (CRTAP; Starman et al., 1989; Barnes et al., 2006; Morello et al., 2006; Valli et al., 2012), prolyl-3-hydroxylase 1 (P3H1/LEPRE1), cyclophillin B (PPIB; Morello et al., 2006; Fratzl-Zelman et al., 2010; Marini et al., 2010; Valli et al., 2012; Zhang et al., 2012), FKBP10 (Barnes et al., 2012), WNT-1 (Pyott et al., 2013), and serpin peptidase inhibitor clade F member 1 (SERPINF1, Homan et al., 2011). In our report, the clinical presentation of $\mathrm{OI}$ in the proband and her mother was mild, and the associated finding of a frameshift mutation in both patients allowed diagnosis of type I osteogenesis imperfecta.

Many patients diagnosed with mild OI type I have null variants in either COL1A1 or COL1A2 genes and a sec-

\section{a)}

CCU GGU CCC CAA GGC UUC CAA GGU CCC CCU GGU GAG CCU GGC GAG CCU GGA GCU UCA GGU CCC AUG GGU CCC CGA GGU CCC CCA GGU CCC CCU GGA AAG AAU GGA GAU GAU GGG 234 GAA GCU GGA AAA CCU GGU CGU CCU GGU GAG CGU GGG CCU CCU GGG CCU CAG GGU GCU CGA GGA UUG CCC GGA ACA GCU GGC CUC CCU GGA 264 AUG AAG GGA CAC AGA GGU UUC

b)

CCU GGU CCC CAA GGC UUC CAA GGU CCC CCU GGU GAG CCU GGC GAG

CCU GGA GCU UCA GGU CCC AUG GGU CCC CGA GGU CCC CCA GGU CCC CCU GGA AAG AAU GGA GAU GAU GGG AAG CUG GAA AAC CUG GUC GUC CUG GUG AGC GUG GGC CUC CUG GGC CUC AGG GUG CUC GAG GAU UGC CCG GAA CAG CUG GCC UCC CUG GAA UGA

Figure 3 - Comparison of mRNA sequences from the wild-type control and proband. The mRNA sequence is shown from nucleotide 586 (codon 196) in exon 9 to nucleotide 810 (codon 270 ) in exon 10 with nucleotide 1 defined as the A of the AUG/ATG translational start codon. The splicing of introns between exon 9 and 10 removed the first $\mathrm{G}$ of a five- $\mathrm{G}$ sequence in the parental cDNA, providing four Gs from nucleotide c.697-700, whereas the proband mutant COL1A1 mRNA only contained a 3-G sequence. This c.700delG genotype induced a frameshift mutation in the proband and her mother. (A) Wild-type COL1A1 mRNA. (B) Proband COL1A1 mRNA with the 700 delG variant. ond allele with the wild-type sequence. The phenotype that results from these null variants is due to approximately $50 \%$ decrease of the wild-type production of the type 1 collagen $\alpha 1$ or $\alpha 2$ chains, known as haploinsufficiency (Redford-Badwal et al., 1996; Slayton et al., 2000; Ben Amor et al., 2013; Peng et al., 2012; Zhang et al., 2012). The phenotypes of patients with haploinsufficiency may be caused by increased degradation of the abnormal type 1 collagen $\alpha$ chain (Garnero et al., 2009), alterations of the mutant mRNA, such as nonsense mediated decay of the mRNA from the mutant allele, or inefficient transport of mRNA to the cytoplasm (Redford-Badwal et al., 1996; Slayton et al., 2000). Nonsense mutations, frameshift mutations, and splice site mutations can induce a premature termination codon in the ORF. For example, 23 of 33 Chinese patients with OI type I had mutations leading to haploinsufficiency in COL1A1; the mutations included eight frameshift mutations, eight splice site mutations, and seven nonsense mutations (Zhang et al., 2012). Mutations associated with haploinsufficiency occurred in exons 2, 3, 5, 7, 9, 11, 17, 18, 19, 24, 28, 31, 36, 42, 43, 46, 47, 49, and 52 (Slayton et al., 2000; Zhang et al., 2012) and introns 44i (Peng et al., 2012). Several of the previously described null mutations of COL1Al appear to be transcribed into (hnRNA) at equivalent levels to wild-type COL1A1, but the mRNA levels of the null mutations are very low or undetectable in the cytoplasm (Slayton et al., 2000).

Similar mutations have been observed less frequently in COL1A2 (Zhang et al., 2012). In a recent study, eighty six Canadians with OI type I due to haploinsufficiency that resulted from nonsense and frameshift mutations showed a risk of 0.62 bone fractures per year which was approximately 95 times greater than that of healthy person of British decent (Ben Amor et al., 2013). The proband and mother described here had suffered only two fractures each in their lifetimes.

A second class of COL1A1 and COL1A2 mutations are translated into procollagen $\alpha$ chains that exhibit altered structure. These mutated procollagen $\alpha$ chains become entwined with normal collagen $\alpha$ chains and the resulting triple helix has a modified configuration, reducing bone strength. The most common mutations result in replacement of one single glycine in the many essential Gly-X-Y triplets in the triple helical region (p.179-1192). The substituted amino acid may include serine, arginine, aspartic acid, valine, or alanine at a single position (Ben Amor et al., 2011; Xu et al., 2011). These point mutations that replace glycine can occur throughout most of the length of COL1A1 and affect collagen stability (Witecka et al., 2008; $\mathrm{Xu}$ et al., 2008; Xiao et al., 2011). These expressed COL1A1 and COL1A2 peptides usually cause more severe OI types (II, III, IV) and usually result from point mutations, insertions, deletions, duplications, and frameshift mutations in the last 200 nucleotides of the reading frame (Xiao et al., 2011). 
Table 1 is a summary of current known mutations of COL1A1 that have been documented. Seven mutations were described in the ninth intron that may reduce the efficiency of the 3' splice site. Interestingly, three patients with the same mutation presented with OI of varying severity (OI type I or type IV). Six mutations were described in exon 10 in two COL1A1 databases. The OI patients with mutations c. $716 \mathrm{G}>\mathrm{A}$, c. $725 \mathrm{G}>\mathrm{A}, \mathrm{c} .740 \mathrm{G}>\mathrm{T}, \mathrm{c} .742 \mathrm{G}>\mathrm{A}$, and c. $743 \mathrm{G}>\mathrm{A}$ were diagnosed with type IV, unclassified OI, OI type I, OI type III, and OI type III, respectively using the osteogenesis imperfecta and Ehlers-Danlos syndrome variant databases (Dalgleish, 1997, 1998) and the UniProKB database. Taken together, these data, combined with the description of the novel mutation c.700delG described here, suggest that additional genes and possibly environmental factors such as nutritional status during development may influence the severity of OI.

If translated, the c.700delG allele would result in a mutant collagen $\alpha 1$ that would be significantly shorter than the wild-type collagen $\alpha 1$ (1464 residues) and would not contain two regions, helix positions p.691-893 and p.910964 , in which single point mutations are usually lethal ((Ben Amor et al., 2011). Because of the mild OI type I symptoms and the dominant inheritance in the pedigree, the COL1A1-c.700delG variant likely leads to haploinsufficiency. The COL1A1-c.700delG was not detected in samples from the proband's maternal grandmother, father, two sisters and was not present in the 50 control samples from unrelated individuals who did not express the COL1A1 mutation.

Limitations of this study include the fact that we only sequenced COL1A1 and COL1A2 genes. While the dominant inheritance pattern suggests involvement of COL1A1 or COL1A2, other known OI candidate genes which induce a recessive OI were not examined, including CRTAP (Barnes et al., 2006; Morello et al., 2006; Valli et al., 2012), P3H1/LEPRE1, PPIB (Morello et al., 2006; Fratzl-Zelman et al., 2010; Marini et al., 2010; Zhang et al., 2012), FKBP10 (Barnes et al., 2012), WNT-1 (Pyott et al., 2013), and SERPINF1 (Homan et al., 2011). It is theoretically feasible that variants in the aforementioned proteins that interact with collagen may compensate for a collagen $\alpha 1$ or $\alpha 2$ variant and thereby contribute to the heterogeneity of the OI symptoms.

Although mutations of COL1A1 and COL1A2 have been associated with OI for several decades, the relationship between the clinical symptoms and location of the variants need further elucidation. Additional genetic or epigenetic factors must influence the variety and severity of symptoms since clinical symptoms of patients with the same mutations can vary among family members (Rauch $e t$ al., 2013). The effect of collagen $\alpha 1$ and $\alpha 2$ variants on proteins that interact with collagen, such as CRTAP, LEPRE1, PPIB, FKBP10, SERPINH1, and SP7 (Garnero et al., 2009; Homan et al., 2011; Ben Amor et al., 2013), would further clarify the mechanisms for the pathology of OI. The novel frameshift variant in exon 10 described here is located only 16 nucleotides upstream of another previously described exon 10 point mutation, c.716G $>\mathrm{A}$, which was detected in a patient with OI type IV (Sarafova et al., 1998). Comparison of the interacting factors for hnRNA, mRNA, and mRNA transport of these two COL1A1 variants may help elucidate the phenotypic differences.

In summary, the COL1A1-c.700delG variant described here in a proband and her mother, if translated, would produce a truncated form of collagen $\alpha 1$ chain without the two essential helical regions (691-893 and 910-964) found in the wild-type form. Because of the mild OI type I

Table 1 - Comparison of OI types with a mutation in the ninth intron or the tenth exon of COL1A1.

\begin{tabular}{|c|c|c|c|c|c|}
\hline \multirow{2}{*}{$\frac{\text { Location }}{09 \mathrm{i}}$} & \multirow{2}{*}{$\begin{array}{c}\text { Mutation } \\
\text { c. } 696+2 \mathrm{~T}>\mathrm{G}\end{array}$} & \multicolumn{2}{|c|}{ Type } & \multirow{2}{*}{$\frac{\text { OI type }}{\text { OI I }}$} & \multirow{2}{*}{$\frac{\text { Reference }}{\text { (Marini et al., 2007) }}$} \\
\hline & & Substitution & Splice site & & \\
\hline $09 \mathrm{i}$ & c. $697-2 \mathrm{~A}>\mathrm{G}$ & Substitution & Splice site & 2 OI I; 1 OI IV & (Hartikka et al., 2004; Marini et al., 2007) \\
\hline $09 \mathrm{i}$ & c. $697-2 \mathrm{~A}>\mathrm{T}$ & Substitution & Splice site & OI I & (Marini et al., 2007) \\
\hline $09 \mathrm{i}$ & c.697-2delA & Deletion & Splice site & OI I & (Lee et al., 2006) \\
\hline $09 \mathrm{i}$ & c.697_2_697-1del & Deletion & Splice site & OI IV; OI I & (Marini et al., 2007; Swinnen et al., 2009) \\
\hline $09 \mathrm{i}$ & c. $697-1 \mathrm{G}>\mathrm{C}$ & Substitution & Splice site & OI I & Ghent, Belgium* \\
\hline $09 \mathrm{i}$ & c.697-1G > T (3) & Substitution & Splice site & OI IV, unknown; OI I & (Marini et al., 2007) Montreal, Canada* \\
\hline 10 & c.700_G-1del & Deletion & Frameshift & OI I & This study \\
\hline 10 & c. $716 \mathrm{G}>\mathrm{A}$ & Substitution & Missense & OI IV & (Marini et al., 2007) \\
\hline 10 & c. $725 \mathrm{G}>\mathrm{A}$ & Substitution & Missense & unknown & (Lee et al., 2006) \\
\hline 10 & c. $740 \mathrm{C}>\mathrm{T}$ & Substitution & Missense & OI I & Valencia, Spain* \\
\hline 10 & c. $742 \mathrm{G}>\mathrm{A}$ & Substitution & Missense & OI III & (Marini et al., 2007) \\
\hline 10 & c. $743 \mathrm{G}>\mathrm{A}$ & Substitution & Missense & OI III & Montreal, Canada* \\
\hline
\end{tabular}

Adapted from the osteogenesis imperfecta and Ehlers-Danlos syndrome variant databases (Dalgleish, 1997; Dalgleish, 1998).

*Location of clinician who submitted the mutation and characteristics of the carrier of the database. 
phenotype, we propose that the novel COL1A1-c.700delG variant likely leads to haploinsufficiency of the collagen $\alpha 1$ chain and that this frameshift variant is the molecular basis of OI type I in this Han Chinese family. Its identification adds further insight to COL1A1 mutations in the Han Chinese population with OI type I.

\section{Acknowledgments}

The authors thank Dr. Weibo Xia (Department of Endocrinology, Key Laboratory of Endocrinology, Ministry of Health, Peking Union Medical College Hospital, Chinese Academy of Medical Sciences, Beijing, China) who provided helpful discussion. The work was supported by the special funds from the Beijing Natural Science Foundation of China (No. 7072073) and the Nursery Fund of General Hospital of Chinese PLA (No. 06MP01).

\section{References}

Barnes AM, Chang W, Morello R, Cabral WA, Weis M, Eyre DR, Leikin S, Makareeva E, Kuznetsova N, Uveges TE, et al. (2006) Deficiency of cartilage-associated protein in recessive lethal osteogenesis imperfecta. $N$ Engl J Med 355:2757-2764.

Barnes AM, Cabral WA, Weis M, Makareeva E, Mertz EL, Leikin S, Eyre D, Trujillo C and Marini JC (2012) Absence of FKBP10 in recessive type XI osteogenesis imperfecta leads to diminished collagen cross-linking and reduced collagen deposition in extracellular matrix. Hum Mutat 33:15891598.

Ben Amor IM, Glorieux FH and Rauch F (2011) Genotypephenotype correlations in autosomal dominant osteogenesis imperfecta. J Osteoporos 2011:540178.

Ben Amor IM, Roughley P, Glorieux FH and Rauch F (2013) Skeletal clinical characteristics of osteogenesis imperfecta caused by haploinsufficiency mutations in COL1A1. J Bone Miner Res 28:2001-2007.

Byers pH and Cole W (2002) Osteogenesis Imperfecta. In: Royce $\mathrm{P}$ and Steinman B (eds) Connective Tissue and its Inheritable Diseases. Wiley-Liss, New York, pp 385-430

Dalgleish R (1997) The human type I collagen mutation database. Nucleic Acids Res 25:181-187.

Dalgleish R (1998) The Human Collagen Mutation Database 1998. Nucleic Acids Res 26:253-255.

Fratzl-Zelman N, Morello R, Lee B, Rauch F, Glorieux FH, Misof BM, Klaushofer K and Roschger P (2010) CRTAP deficiency leads to abnormally high bone matrix mineralization in a murine model and in children with osteogenesis imperfecta type VII. Bone 46:820-826.

Garnero P, Schott AM, Prockop D and Chevrel G (2009) Bone turnover and type I collagen C-telopeptide isomerization in adult osteogenesis imperfecta: associations with collagen gene mutations. Bone 44:461-466.

Glorieux FH, Rauch F, Plotkin H, Ward L, Travers R, Roughley P, Lalic L, Glorieux DF, Fassier F and Bishop NJ (2000) Type $\mathrm{V}$ osteogenesis imperfecta: A new form of brittle bone disease. J Bone Miner Res 15:1650-1658.

Glorieux FH, Ward LM, Rauch F, Lalic L, Roughley PJ and Travers R (2002) Osteogenesis imperfecta type VI: a form of brittle bone disease with a mineralization defect. J Bone Miner Res 17:30-38

Hartikka H, Kuurila K, Körkkö J, Kaitila I, Grénman R, Pynnönen S, Hyland JC and Ala-Kokko L (2004) Lack of correlation between the type of COL1A1 or COL1A2 mutation and hearing loss in osteogenesis imperfecta patients. Hum Mutat 24:147-154.

Homan EP, Rauch F, Grafe I, Lietman C, Doll JA, Dawson B, Bertin T, Napierala D, Morello R, Gibbs R, et al. (2011) Mutations in SERPINF1 cause osteogenesis imperfecta type VI. J Bone Miner Res 26:2798-2803.

Lee KS, Song HR, Cho TJ, Kim HJ, Lee TM, Jin HS, Park HY, Kang S, Jung SC and Koo SK (2006) Mutational spectrum of type I collagen genes in Korean patients with osteogenesis imperfecta. Hum Mutat 27:599.

Marini JC, Forlino A, Cabral WA, Barnes AM, San Antonio JD, Milgrom S, Hyland JC, Körkkö J, Prockop DJ, De Paepe A, et al. (2007) Consortium for osteogenesis imperfecta mutations in the helical domain of type I collagen: regions rich in lethal mutations align with collagen binding sites for integrins and proteoglycans. Hum Mutat 28:209-221.

Marini JC, Cabral WA and Barnes AM (2010) Null mutations in LEPRE1 and CRTAP cause severe recessive osteogenesis imperfecta. Cell Tissue Res 339:59-70.

Morello R, Bertin TK, Chen Y, Hicks J, Tonachini L, Monticone M, Castagnola P, Rauch F, Glorieux FH, Vranka J, et al. (2006) CRTAP is required for prolyl 3-hydroxylation and mutations cause recessive osteogenesis imperfecta. Cell 127:291-304.

Moul A, Alladin A, Navarrete C, Abdenour G and Rodriguez MM (2013) Osteogenesis imperfecta due to compound heterozygosity for the LEPRE1 gene. Fetal Pediatr Pathol 32:319325.

Pallos D, Hart PS, Cortelli JR, Vian S, Wright JT, Korkko J, Brunoni D and Hart TC (2001) Novel COL1A1 mutation (G559C) [correction of G599C] associated with mild osteogenesis imperfecta and dentinogenesis imperfecta. Arch Oral Biol 46:459-470.

Peng H, Zhang Y, Long Z, Zhao D, Guo Z, Xue J, Xie Z, Xiong Z, $\mathrm{Xu} \mathrm{X}, \mathrm{Su} \mathrm{W}$, et al. (2012) A novel splicing mutation in COL1A1 gene caused type I osteogenesis imperfecta in a Chinese family. Gene 502:168-171.

Pyott SM, Tran TT, Leistritz DF, Pepin MG, Mendelsohn NJ, Temme RT, Fernandez BA, Elsayed SM, Elsobky E, Verma I, et al. (2013) WNT1 mutations in families affected by moderately severe and progressive recessive osteogenesis imperfecta. Am J Hum Genet 92:590-597.

Rauch F and Glorieux FH (2005) Osteogenesis imperfecta, current and future medical treatment. Am J Med Genet C Semin Med Genet 139C:31-37.

Rauch F, Moffatt P, Cheung M, Roughley P, Lalic L, Lund AM, Ramirez N, Fahiminiya S, Majewski J and Glorieux FH (2013) Osteogenesis imperfecta type V: marked phenotypic variability despite the presence of the IFITM5 c.-14C $>$ T mutation in all patients. J Med Genet 50:21-24.

Redford-Badwal DA, Stover ML, Valli M, McKinstry MB and Rowe DW (1996) Nuclear retention of COL1A1 messenger RNA identifies null alleles causing mild osteogenesis imperfecta. J Clin Invest 97:1035-1040.

Sarafova AP, Choi H, Forlino A, Gajko A, Cabral WA, Tosi L, Reing CM and Marini JC (1998) Three novel type I collagen 
mutations in osteogenesis imperfecta type IV probands are associated with discrepancies between electrophoretic migration of osteoblast and fibroblast collagen. Hum Mutat 11:395-403.

Sillence DO, Senn A and Danks DM (1979) Genetic heterogeneity in osteogenesis imperfecta. J Med Genet 16:101-116.

Slayton RL, Deschenes SP and Willing MC (2000) Nonsense mutations in the COL1A1 gene preferentially reduce nuclear levels of mRNA but not hnRNA in osteogenesis imperfecta type I cell strains. Matrix Biol 19:1-9.

Starman BJ, Eyre D, Charbonneau H, Harrylock M, Weis MA, Weiss L, Graham Jr JM and Byers pH (1989) Osteogenesis imperfecta. The position of substitution for glycine by cysteine in the triple helical domain of the pro alpha 1(I) chains of type I collagen determines the clinical phenotype. J Clin Invest 84:1206-1214.

Swinnen FK, De Leenheer EM, Coucke PJ, Cremers CW and Dhooge IJ (2009) Audiometric, surgical, and genetic findings in 15 ears of patients with osteogenesis imperfecta. Laryngoscope 119:1171-1179.

Valli M, Barnes AM, Gallanti A, Cabral WA, Viglio S, Weis MA, Makareeva E, Eyre D, Leikin S, Antoniazzi F, et al. (2012) Deficiency of CRTAP in non-lethal recessive osteogenesis imperfecta reduces collagen deposition into matrix. Clin Genet 82:453-459.

van Dijk FS, Byers PH, Dalgleish R, Malfait F, Maugeri A, Rohrbach M, Symoens S, Sistermans EA and Pals G (2012) EMQN best practice guidelines for the laboratory diagnosis of osteogenesis imperfecta. Eur J Hum Genet 20:11-19.

Ward LM, Rauch F, Travers R, Chabot G, Azouz EM, Lalic L, Roughley PJ and Glorieux FH (2002) Osteogenesis imperfecta type VII: an autosomal recessive form of brittle bone disease. Bone 31:12-18.

Witecka J, Augusciak-Duma AM, Kruczek A, Szydlo A, Lesiak M, Krzak M, Pietrzyk JJ, Männikkö M and Sieron AL (2008) Two novel COL1A1 mutations in patients with osteogenesis imperfecta (OI) affect the stability of the collagen type I triple-helix. J Appl Genet 49:283-295.
Xiao J, Cheng H, Silva T, Baum J and Brodsky B (2011) Osteogenesis imperfecta missense mutations in collagen: structural consequences of a glycine to alanine replacement at a highly charged site. Biochemistry 50:10771-10780.

Xu K, Nowak I, Kirchner M and Xu Y (2008) Recombinant collagen studies link the severe conformational changes induced by osteogenesis imperfecta mutations to the disruption of a set of interchain salt bridges. J Biol Chem 283:3433734344.

Xu Z, Li Y, Zhang X, Zeng F, Yuan M, Liu M, Wang QK and Liu JY (2011) Identification and molecular characterization of two novel mutations in COL1A2 in two Chinese families with osteogenesis imperfecta. J Genet Genomics 38:149156.

Zhang ZL, Zhang H, Ke YH, Yue H, Xiao WJ, Yu JB, Gu JM, Hu WW, Wang C, He JW, et al. (2012) The identification of novel mutations in COL1A1, COL1A2, and LEPRE1 genes in Chinese patients with osteogenesis imperfecta. J Bone Miner Metab 30:69-77.

\section{Internet Resources}

Osteogenesis imperfecta and Ehlers-Danlos syndrome variant databases compiled of previously reported OI mutations in COL1A1 and COL1A2, http://www.le.ac.uk/ge/collagen/; last update Mar 13, 2013.

UniProKB database, http://www.uniprot.org/uniprot/P02452.

ORF Finder in BLAST software suite, http://www.ncbi.nlm.nih.gov/gorf/.

\section{Supplementary Material}

The following online material is available for this article:

- Table S1 - Clinical, biochemical and inheritance characteristics of OI types.

This material is available as part of the online article from http://www.scielo.br/gmb.

Associate Editor: Maria Luiza Petzl-Erler

License information: This is an open-access article distributed under the terms of the Creative Commons Attribution License, which permits unrestricted use, distribution, and reproduction in any medium, provided the original work is properly cited. 